\title{
Alocação de Recursos em Funções de Redes Virtualizadas: Desafios e Perspectivas Aplicado em Data enter
}

\author{
Rafael Souza $^{1}$, Marcelo Santos ${ }^{2}$, Stênio Fernandes ${ }^{1}$ \\ ${ }^{1}$ Centro de Informática - Universidade Federal de Pernambuco (UFPE) \\ Caixa Postal 181 - CEP 89802-112 - Recife - PE - Brasil \\ \{rrs4, sflf\}@cin.ufpe.br \\ ${ }^{2}$ Instituto Federal do Sertão Pernambucano (IF Sertão-PE) \\ Caixa Postal 508 - CEP 56000-000 - Salgueiro -PE - Brasil. \\ marcelo.santoseifsertao-pe.edu.br
}

\begin{abstract}
The implementation of a Network Function Virtualization (NFV) denotes a widespread shift in communication and network services from a service provider network to a Cloud-based network. Its use has the potential to provide Cloud-based network services with lower operating and capital costs. In this context, This work aims to identify and discuss NFV applications in data center architectures. This study reviews of the literature to raise the main research on resource allocation of NFV in the data center. From this research, we have identified evidence on the problems and solutions associated with the resources allocation of NFV in data centers. Finally, we identify gaps, open challenges that can be used to target future research. Finally, we identify gaps, open challenges that can be used to direct future research.
\end{abstract}

Resumo. A implementação de uma Network Function Virtualization (NFV) denota uma mudança generalizada nos serviços de comunicação e rede de uma rede de provedores de serviços para uma rede baseada em nuvem. Seu uso tem o potencial de fornecer serviços de rede baseados em nuvem com menores custos operacionais e de capital. Neste contexto, este trabalho visa identificar e discutir aplicações de NFV em arquiteturas de data center. Este estudo realiza uma revisão da literatura, para levantar as principais pesquisas sobre alocação de recurso de NFV no data center. A partir dessa pesquisa, foi identificado evidências sobre os problemas e soluções associadas a alocação de recursos de NFV em data centers. Finalmente, identificamos lacunas, desafios abertos que podem ser usadas para direcionar pesquisa futura.

\section{Introdução}

A virtualização emergiu como uma paradigma que permite virtualizar funções de redes que executavam em hardwares dedicados em máquinas de propósito geral através do uso de tecnologias já aplicadas em computação em nuvem e data center. Dessa forma, é possível, por exemplo, realizar a automatização de processos, com redução de custos operacionais (Operational Expenditure - OPEX) e melhor eficiência nos gastos de capital (Capital Expenditure - CAPEX). Nessa perspectiva, surgiram as redes definidas por software (Software Defined Networking - SDN) que permitem desacoplar o plano de 
controle (lógico) do plano de dados, propiciando um cenário evolutivo das redes atuais [Feamster et al. 2013], bem como a Virtualização de Funções de Redes (Network Function Virtualization - NFV) que foi criada pelo Instituto Europeu de Normas de Telecomunicações (ETSI) [Schaffrath et al. 2009] [Chiosi et al. 2012]. Ambas as tecnologias vem sendo utilizadas para dinamicidade e flexibilidade do gerenciamento de redes no data center.

Poder criar funções de rede virtualizadas (Virtualized Network Function - VNF) permite uma maior flexibilidade de uma rede baseada em software sobre uma infraestrutura física. A ideia de arquitetura NFV permite virtualizar funções de rede como, por exemplo, firewall e balanceadores de carga. Além disso, NFV permite organizar os recursos físicos e virtuais otimizando o provisionamento, configuração de rede, alocação de largura de banda, automação de operações, monitoramento, segurança e políticas de controle. A capacidade de virtualização permite ainda trabalhar com arquitetura de rede tradicional para reduzir os custos da empresa na aquisição de novo hardware de rede. Estudos mostram ainda que NFV poderá reduzir as despesas de energia e espaço para alocação de novas máquinas [Rosa et al. 2015].

Uma pesquisa realizada em 2015 pela IHS Markit sobre o crescimento do NFV em 2015-2019, projetou um investimento próximo de US \$11,6 bilhões. O mesmo estudo afirma que $100 \%$ dos prestadores de serviços consultados pretendem adaptar suas infraestruturas para suportar NFV. Desses, 81\% esperam fazê-lo até 2017 [IHS Markit 2015]. No cenário específico de data centers (data centers), a adoção do NFV transfere funções de rede para um hardware comercial off-the-shelf (COTS), em vez de dispositivos proprietários especializados. Nesse cenário, a padronização de protocolos permite que o hardware de propósito geral seja utilizado para reduzir custos, ganhando simultaneamente flexibilidade através da interoperabilidade de diferentes sistemas.

A infraestrutura de data center é um serviço especializado de valor agregado, que fornece capacidades de processamento e armazenamento de dados escaláveis para organizações de qualquer tamanho, como Amazon, Google, Facebook e Yahoo. Essas organizações investem profundamente no planejamento de data center para evitar, por exemplo, picos de inatividade ou sobrecarga inesperados. Além dos custos diretos do tempo de inatividade do sistema, há mais custos intangíveis, como reputação danificada, menor satisfação dos funcionários e redução da retenção de clientes. Um estudo conduzido pelo Instituto Ponemon descobriu que o custo médio do tempo de inatividade do data center está perto de US $\$ 9.000$ por minuto [Ponemon Institute 2016]. Assim, é importante fornecer serviços e capacidades analíticas com atrasos mínimos. Dessa forma, o design e o desempenho de data center em larga escala, bem como o gerenciamento de ativos de TI, não podem ser negligenciados. Assim, uma revisão da literatura de NFV em data center torna-se necessária para identificar os avanços e desafios, bem como quais técnicas de outras áreas podem ser aplicadas em NFV no cenário de um data center.

No cenário de NFV e data center, a maioria das abordagens atuais enfocam o gerenciamento do data center para interconectar os recursos virtuais com a estrutura física [Maini and Manzalini 2014] [Riccobene et al. 2014][Shen et al. 2014] [Clayman et al. 2014]. Outras pesquisas, estão relacionadas com o desempenho do NFV [Schaffrath et al. 2009] [Wang et al. 2014]. Considerando o desempenho, os requisitos de gestão no ambiente NFV devem ter a capacidade de suportar variações de carga de 
trabalho. Assim, um dos desafios da gestão no NFV no data center em computação nas nuvens é a demanda que varia de um dia para outro ou mesmo por hora. Essa flutuação torna difícil gerenciar o processo manualmente. Uma possível solução é dividir Máquinas Virtuais (Virtual Machine - VM) em diferentes nós ou clusters. Há muitos aspectos do NFV a analisar, desde os mais concretos até os mais abstratos, como máquinas virtuais e links virtuais. Assim, este trabalho está interessado em investigar os esforços específicos na alocação de recurso de NFV em arquiteturas de data center.

Dentro da IETF/IRTF, o Network Function Virtualization (NFVRG) investiga NFV sob várias perspectivas com o objetivo de identificar as abordagens que podem ser definidas, implementadas e utilizadas no curto prazo, além de também identificar os futuros desafios de pesquisa. Em particular, as principais áreas de interesse incluem soluções: virtualização, alocação e recurso e encadeamento de funções de rede (Service Function Chain - SFC), bem como identificação de futuros desafios de pesquisa na área. Outro grupo relacionado com este trabalho é Benchmarking Methodology Working Group (BMWG), pesquisa por trabalhos relacionados também com melhorias de desempenho, entre os quais verificam-se trabalhos com foco específico em data center [Samuel and Rapp 2017][Avramov and Rapp 2016]. Assim, acreditamos que os desafios de pesquisas encontradas podem ser uma contribuição para o grupo NFVRG, que tem investigado, por exemplo, a performance de data center [Samuel and Rapp 2017] [Avramov and Rapp 2016], virtualização de redes [Zúñiga et al. 2017], Alocações de Recursos [Szabo et al. 2016], encadeamento de serviço [Do and Kim 2017], bem como identificação de futuros desafios de pesquisa para a comunidade da IETF/IRTF. Dessa forma, temos como contribuição a definição de desafios e possíveis soluções de NFV aplicadas ao ambiente de data center. Além disso, espera-se como resultado contribuir para o Draft da Internet (I-D) entitulado "Gap Analysis on Network Virtualization Activities " ". A organização deste artigo é a seguinte: Fundamentos e Aspectos Teóricos (Seção 2), Desafios e Oportunidades seção (Seção 3), Considerações Finais (Seção 4).

\section{Fundamentos e Aspectos Teóricos}

\subsection{Virtualização de Rede}

Ao utilizar máquinas de propósito geral (COTS) em uma infraestrutura NFV, podemos executar de forma virtualizada diversas funções de rede distintas, tais como rede privada virtual (Virtual Private Network - VPN), firewall, balanceador de carga, rede de distribuição de conteúdo (Content Delivery Network - CDN), subsistema multimídia IP (Internet Protocol Multimedia Subsystem - IMS), Roteador, entre outros. Como tal, as funções de rede virtualizadas, também chamadas de Função de Rede Virtual (Virtualized Network Function - VNF) são instanciadas dentro da rede sob demanda. Requisitos de desempenho são identificados nas aplicações do cliente ETSI [ETSI 2014] e as quatro partes da arquitetura de referência NFV são mostradas na Figura 1

- VNF é uma Máquina Virtual (Virtual Machine - VM) destinada a executar uma determinada função de rede. Uma VNF é comumente administrada por um sistemas simples de gerenciamento de elementos (Element Management - EM), que permite a criação, configuração, monitoramento, segurança, confiabilidade e desempenho;

\footnotetext{
${ }^{1}$ https://tools.ietf.org/html/draft-bernardos-nfvrg-gaps-network-virtualization-04
} 

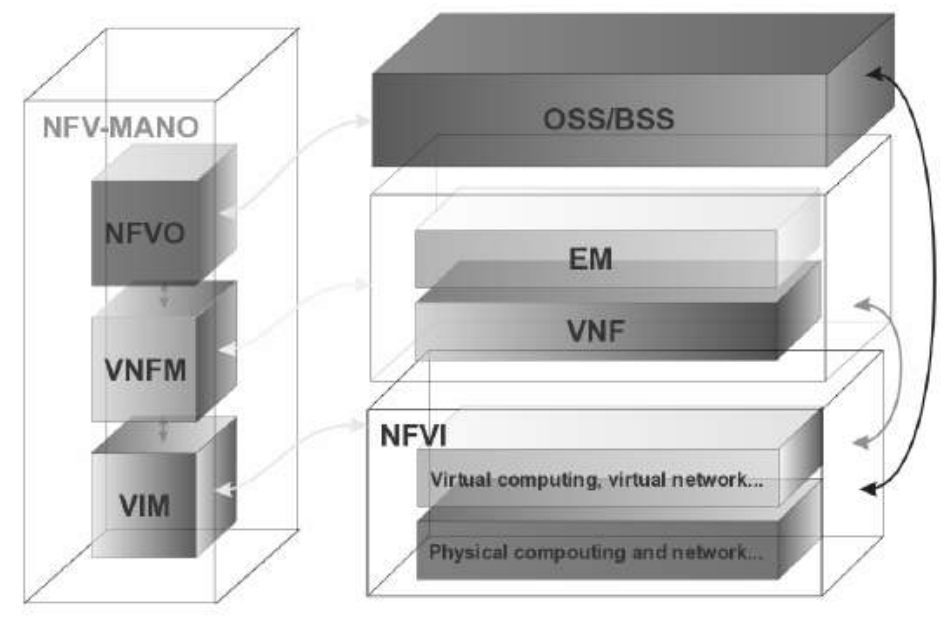

Figura 1. Arquitetura de Referência NFV. Adaptado de [ETSI 2013].

- Infraestrutura para Virtualização das Funções da Rede (NFV infrastructure NFVI), consiste em hardware e software, que é a base para o ambiente virtualizado através de um Hypervisor;

- Suporte Operacional (Operational Support Systems - OSS) e Sistems de Suporte ao Negócio (Business Support Systems - BSS) lida com o sistema legado que precisa se integrar com o ambiente NFV para se adequar aos processos da operadora

- Gerenciamento e Orquestração (Management and Orchestration - MANO) gerenciam o ciclo de vida de recursos físicos, VNFs e software.

O Gerenciador de Funções de Rede Virtual (VNF Manager - VNFM), gerência o ciclo de vida de VNFs sob controle NFVO, (ii) o VIM gerência a infraestrutura de uma solução baseada em NFV e (iii) O NFV Orchestrator (NFV Orchestrator - NFVO), concentra a inteligência para a distribuição de VNFs ao longo da rede. O NFVO também é responsável por ligar os ambientes físicos e virtuais, bem como a integração com OSS/BSS.

\subsection{Infraestrutura do Data Center}

$\mathrm{O}$ data center é um conjunto de recursos escaláveis para processamento e armazenamento de dados para organizações de qualquer tamanho, como empresas, instituições educacionais, indústrias, agências governamentais, entre outros. Além disso, um sistema de data center genérico (Figura 2), consiste essencialmente nos seguintes subsistemas: (i) infraestrutura de TI; (ii) infraestrutura de refrigeração; e (iii) infraestrutura de energia. [Maini and Manzalini 2014].

- A infraestrutura típica de TI consiste em três componentes de hardware principais: (i) os servidores usam Ethernet para conectar sistemas cruzados de acesso a arquivos remotos através de um dispositivo de armazenamento conectado a uma rede (Network-Attached Storage - NAS); (ii) armazenamento refere-se a dispositivos de gravação de dados conectados tipicamente através da rede de área de armazenamento (Storage Area Network - SAN); e (iii) equipamento de dispositivos de rede, tais como switches e roteadores; 
2 Cooling Infrastructure

\section{Power Infrastructure}
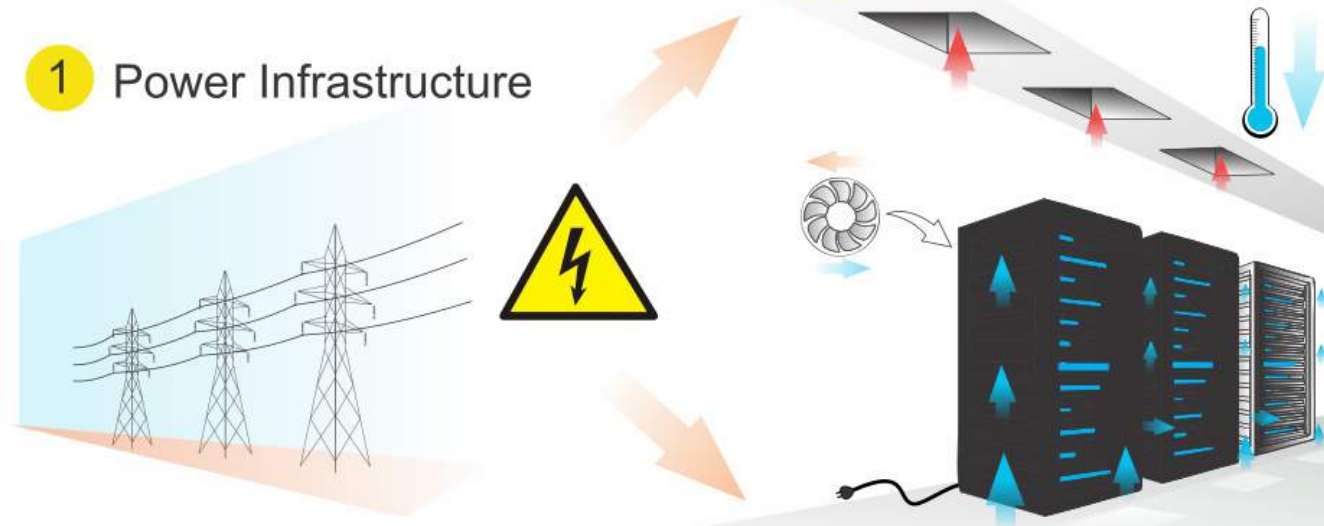

3 IT Infrastructure

Figura 2. Infraestrutura do data center.

- A infraestrutura de energia fornece alimentação ininterrupta na frequência e tensão especificadas para os dispositivos de TI e refrigeração [Fan et al. 2007]. Normalmente, a energia passa através de transformadores (Step Down Transformers SDT), unidades de distribuição de energia (Power Distribution Units - PDU), interruptores estáticos ou automáticos, fontes de alimentação ininterrupta (Power Supplies - UPSs) e, finalmente, barras de alimentação do rack são usados para conectar os dispositivos elétricos. O UPS fornece energia e backup através de baterias em caso de falhas de curto prazo. Considerando que outro fornecimento local de energia (por exemplo, eletricidade, diesel, energia renovável e energia nuclear) fornece energia para interrupções de tempo mais longas;

- A infraestrutura de refrigeração inclui unidades de ar condicionado (Computer Room Air Conditioning - CRAC), e as Torres de resfriamento (cooling towers) [Arregoces and Portolani 2003]. A infraestrutura de refrigeração representa aproximadamente $15-20 \%$ em média do consumo de energia do data center [Miller 2011].

\subsection{Dependabilidade em Data Center}

Devido à crescente expansão das virtualizações de redes de computadores, é fundamental que se faça a análise das mesmas sob as métricas de dependabilidade, que são importantes para o avanço tecnológico de NFV em data center, de forma a determinar se uma aplicação de rede é confiável. Os primeiros conceitos de dependabilidade foram inicialmente definidos no artigo Dependability Basic Concepts and Terminology [Avizienis et al. 2004] e desde então, têm sido largamente utilizados tanto na academia como na indústria. As métricas são compostas da seguinte forma:

- Disponibilidade é a operação contínua independente das falhas do sistema; 
- Confiabilidade é o correto contínuo desempenho da rede, sem a presença de falhas;

- Integridade é a situação em que não há corrupção de dados no tempo;

- Segurança é a correta detecção de falhas do sistema;

- Manutenção é a facilidade para corrigir o sistema em resposta a uma falha.

Uma distinção importante é feita entre prevenção de fault, tolerância, previsão (forecasting) e remoção. O estresse é colocado em particular na tolerância, ou seja, a capacidade de tolerar falhas durante a execução do sistema. A Figura 3 mostra uma relação entre os tipos de falhas, que são consideradas faults físicos ou imperfeições que levam, possivelmente, a failure (no sentido do comportamento esperado). O trânsito de faults para failures é normalmente conchecido como o desvios do comportamento esperado, chamado erros. Failures, se não tratadas ou solucionadas, podem por sua vez, desencadear outras faults do sistema e levar as operações do data center, de forma cíclica, a um desastre incontrolável.

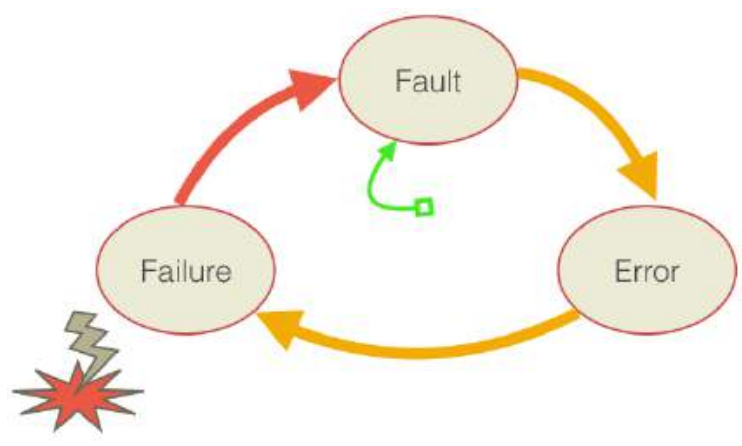

Figura 3. Tipos de Falhas.

Os problemas de falhas de redes relacionados com alocação de recurso, requerem abordagens sistemáticas, visando à melhoria da qualidade do sistema em termos de NFV aplicados ao data center, tolerância a erros e ocorrência de falhas, verificação do funcionamento correto, validação das funcionalidades e da missão do sistema. Para tal, listamos a seguir alguns desafios em abertos, seguindo as categorias de falhas de acordo com [Avizienis et al. 2004]:

- Prevenção de Defeitos: tornou-se fator crítico no gerenciamento da sobrevivência de redes no data center. O impacto da falha é agravado por volumes extremamente elevados de tráfego transportados em redes no data center e, nesse aspecto, torna-se um desafio a inserção da prevenção de falha de VNF em data center. A prevenção assegura a proteção em relação às falhas de entrada em tempo de execução, permitindo prevenção contra sua ocorrência e seus efeitos. Uma das formas que vêm sendo investigados nas áreas de computação nas nuvens, mas um dos desafios que estão relacionados é a aplicação de técnicas de injeção de falhas [Kooli and Di Natale 2014].

- Tolerância a Falhas: a aplicação de NFV tolerante a falha no data center é baseada na hipótese de que falhas existem no sistema e eventualmente serão ativadas, causando erros. Os efeitos de falhas devem ser planejados para recuperação da função de rede, de forma a reduzir o tempo de reparo ou o período em que a rede ficará inativa. A falha de um elemento de rede pode levar a um grande impacto na 
rede de substrato, o que, por sua vez, leva à perda de dados, que pode interromper serviços de comunicação. Alguns exemplos são a melhoria dos processos de planejamento da rede, pois, para uma função de rede, o uso de tais técnicas é um passo necessário para sistemas confiáveis [Salfner et al. 2010].

- Remoção de Defeitos: para remover as falhas durante o desenvolvimento do sistema (por exemplo, verificação, validação) ou durante a sua utilização. Em particular, a remoção de falhas durante o uso do sistema pode ser implementada como manutenção corretiva, na qual as falhas são encontradas e corrigidas ou manutenção preventiva, para descobrir falhas existentes antes que elas se transformem em erros.

- Previsão de Defeitos: analisa o comportamento do sistema de forma qualitativa ou quantitativa, em relação à ocorrência ou ativação de falha. Na prática, o objetivo é avaliar o comportamento do sistema a fim de estimar as consequências futuras de uma falha usando modelagem de sistema ou teste de sistema. A predição de falha é particularmente útil no contexto de falhas residuais, isto é, falhas que escaparam ao processo de teste, e que não podem ser toleradas por outros mecanismos de tolerância a falhas [Vieira et al. 2009]. Um preditor de falhas on-line prevê falhas de entrada em tempo de execução, principalmente usando dados passados do sistema, usados para treinar o preditor e informações sobre o estado atual do sistema obtido por meio do monitoramento de variáveis do sistema.

\section{Requisitos e Desafios de NFV em Data Center}

O ETSI define que uma rede de substrato como entidades compostas por um número de VNFs [ETSI 2014], assim, uma função de rede (um nó) pode ser implementada via VNF. Portanto, ao ser enviado um pacote de um computador de origem para um destino, o mesmo passar por um conjunto de VNFs para fazer parte do serviço de rede oferecido. Provisionar corretamente a quantidade de recursos dentro de uma infraestrutura de um data center é um desafio, pois, segundo o Instituto Ponemon, em 2016 houve um aumento de $38 \%$ nos custos de inatividade do data center em comparação a 2010, resultando em uma perda financeira de US\$740.357 [Ponemon Institute 2016]. Nesse sentido, o processo de alocação eficiente de recursos torna-se essencial diante de um cenário dinâmico e com alta demanda de recursos.

\subsection{Alocação de Recursos em Data Center e SLA}

Para caracterizar o problema de alocação de recursos para um ambiente de data center em nuvem, o pesquisador de [Liu and Wood 2015] constrói um Data Plane Development Kit (DPDK). O DPDK baseia-se numa estrutura de monitorização e análise em larga escala de redes de alta velocidade. Após a aquisição dos dados, os mesmos são enviados para um processamento que é baseado no Apache Storm, que é uma ferramenta de análise de dados em tempo real.

Os trabalhos de [Rankothge et al. 2015] [Moens and De Turck 2014] abordam o problema da alocação de recursos com cadeia de serviços e otimização através da implementação de algoritmos genéticos e de progração linear inteira (Integer Linear Programming - ILP) integrados ao emulador Mininet com o controlador Ryu. Uma estratégia de posicionamento de Virtual data center - (VDC) com melhor reconhecimento de rede, reduz o impacto da carga de trabalho na infraestrutura de comunicação física. Outro 
aspecto identificado por [Moens and De Turck 2014] é a criação de um algoritmo ILP, através das ferramentas SCALA e CPLEX. O algoritmo ILP aloca recursos VNF em um ambiente NFV. Por essa razão, considera-se um cenário híbrido, no qual um hardware físico dedicado fornece parte dos serviços.

Para satisfazer o acordo de nível de serviço (Service Level Agreement - SLA) dos usuários em uma rede de data center definida por software, o estudo [Huang et al. 2015] investiga os problemas das cadeias de serviços e da orquestração. O objetivo é criar algoritmos ILP para avaliar o desempenho da orquestração de múltiplas cadeias de serviço NFV. O problema de escala foi investigado por [Xia et al. 2015] [Soares and Sargento 2015] [Callegati et al. 2016]. Em [Xia et al. 2015], foca em maximizar a qualidade do desempenho do serviço, avaliou a percepção do usuário no tocante à rede de ponta a ponta e desempenho lado a lado do sistema. A fim de investigar o monitoramento de recursos em pequenas escalas de tempo, [Soares and Sargento 2015] avaliou os requisitos de qualidade de experiência (Quality of Experience - QoE). Esta análise foi baseada em opiniões de convidados e host sobre "uso" e "carga" para a análise de variância. O estudo [Callegati et al. 2016], utilizou um cenário NFV para identificar limites potenciais e reais do desempenho de virtualização de rede em infraestruturas em nuvem OpenStack.

\subsection{Mapeamento de Cadeia de Serviço em Data Center}

O IETF lançou um grupo para a arquitetura da encadeamento de funções de rede (Service Function Chain Working Group - SFC-WG) [Quinn and Halpern 2014] para desacoplar a topologia física e os funcionamentos da função de serviço para que a reconfiguração do serviço possa ser simplificada. Alguns empresas como Cisco e Huawei Inc. promoveram esta arquitetura em uma fase de padronização [Aldrin et al. 2016]. Outros trabalhos mais recente investiga a aplicação de SFC em data center [Kumar et al. 2017]. No entanto, observamos nesta revisão da literatura que um dos gap identificado, podem ser futuros desafios de pesquisa na comunidade do IETF/IRTF, é o mapeamento de cadeia de serviço em data center.

O problema encontrado nas redes virtuais embutidas (Virtual Network Embedded - VNE), consiste no mapeamento de um conjunto de requisições de VNF (VNF requests ou VNRs), sobre uma rede de substrato do data center, de forma eficiente e que garanta a maior quantidade de requisições atendidas. Os problemas que envolvem o mapeamento de cadeia de serviço são complexos porque as cadeias incluem serviços de redes tradicionais. Além disso, encadeamento pode ser construído manualmente através de uma interface de usuário ou dinamicamente através de algoritmos. Assim, a flexibilidade é introduzida por meio da virtualização de forma dinâmica de cadeias de VNF, para implantar estrategicamente, um conjunto de nós de rede física, de modo a alcançar o objetivo de um operador predefinido ou atender a requisitos de SLA. No entanto, a atual rede estática de cadeias de posicionamento depende da localização física.

O mapeamento de cadeia de serviço concentra-se em como alocar os VNF nas infraestruturas de rede de forma adequada, considerando um conjunto de serviços de rede solicitados. Além disso, a otimização de recursos deve ser realizada com relação a um objetivo específico, por exemplo, maximização dos recursos de rede remanescentes, minimização do consumo de energia do data center. O mapeamento de SFC de VNFs 
para um data center é composto de um serviço de rede fim a fim. O SFC é composto pelo conjunto ordenado de VNFs que a rede de substrato executa para cumprir atributos do serviço em quesitos de dependabilidade. No entanto, mesmo tendo-se o conhecimento prévio de todas as requisições de redes virtuais, o mapeamento ótimo é um problema NPdifícil [Amaldi et al. 2016], onde a maioria dos trabalhos realizados nessa área, tem se concentrado no desenho de algoritmos heurísticos ou meta-heurísticos e no uso de redes com complexidade mínima ao resolver modelos de ILP.

Neste contexto, a aplicação de estratégias de mapeamento de NFV em data center, tendo como entrada o SFC para alocação de recurso, é uma lacuna de pesquisa para alocação das funções de rede, isso porque a mudança de SFC é dinâmica e as topologias do data center são tipicamente homogêneas, o que significa que os servidores têm a mesma capacidade de computação, armazenamento e comunicação. Para tal, podemos projetar mecanismos que melhorem o desempenho do data center. Nesse sentido, o desempenho da rede de substrato, será afetado pelo comportamento das diferentes funções de composição e pela ordem em que as funções são processadas. Portanto, é primordial conseguir um encadeamento de cadeia de serviços eficiente em relação aos objetivos do design da rede. Entre as perguntas não respondidas, podemos citar:

- Como concatenar os diferentes VNFs eficientemente para compor uma rede física do data center de maneira mais adequada?

- Como desenvolver algoritmos exatos e heurísticos, a fim de reduzir os atrasos na rede devido ao congestionamento por meio de SFC no data center?

- Qual o nível de confiabilidade, desempenho e sustentabilidade que NFV deve garantir no data center, com o mapeamento de SFC?

- Como o NFV ajudará o provedor de data centers a agregar e analisar índices de redundância?

- Como otimizar um mapeamento de recuperação SFC em situação de desastres no data centers?

- Como projetar o NFV em data center, com integração, granularidade, tempo de implantação, gerenciamento, manutenção e operações sustentáveis?

- Como considerar a latência mínima do data center ao distribuir as VNF geograficamente?

- Como economizar energia em data center durante a replicação de rede VNF ao mesmo tempo que maximiza a disponibilidade?

- Como construir, de forma eficiente, um SFC adequado em relação aos objetivos do operador?

- Como mapear VNF na rede de substrato do data center, considerando o mapeamento de nós e links?

- Como fazer um mapeamento eficiente requisições de rede virtual em uma rede de substrato compartilhada no data centers?

\subsection{Escalonamento de VNF em Data Center}

Um dos objetivo do escalonamento de recursos é identificar aqueles que são adequados, e em seguida realizar escalonamento das cargas de trabalho apropriadas, no tempo, e maximizar a utilização dos recursos disponíveis. O escalonador não só tem total informação acerca de todas as tarefas que estão em execução, pendente e utilização dos recursos, mas também gerência a fila de tarefas e o conjunto de recursos. Assim, pode facilmente prever 
o comportamento dos recursos, e é capaz de atribuir tarefas aos recursos, de acordo com determinados requisitos de QoS, como a utilização de CPU, disponibilidade, confiabilidade, a fim de manter um nível de qualidade de serviço necessário ou ainda minimizar o tempo de conclusão da carga de trabalho. Assim, torna-se importante ter um eficiente mapeamento da carga de trabalho de recursos, antes do processo de escalonamento [Singh and Chana 2016].

O problema de escalonamento em uma rede de data center tradicional têm muitos aspectos específicos ligados ao desenvolvimento de algoritmos, que são diferentes do padrão router escalonamento. Por exemplo, em um roteador ou switch projetado para operar em um chassi ou rack pode empregar um escalonamento centralizado para calcular uma correspondência entre os módulos de entrada e de saída em cada intervalo de tempo de transmissão [McKeown et al. 1999]. No entanto, em uma aplicação de NFV em data center, o problema de escalonamento procura auxiliar na melhoria da execução de cada função para minimizar o tempo de execução total sem degradar o desempenho do serviço e respeitando todas as precedências e dependências entre os VNFs que compõem a rede de substrato. Isso porque, normalmente, a execução VNFs deve ser realizada a fim de minimizar o tempo total de execução dos serviços de rede e, assim, obter melhor desempenho.

Na terminologia NFV, a economia de recursos é obtida por alocação de recursos sob demanda. Além disso, para aumentar a utilização de recursos, é possível usar técnicas de programação para permitir que os VNFs compartilhem o recurso. Assim, o problema escalonamento de recursos de NFV em data center é um trabalho desafiador, pois, para cargas de trabalho em data center, depende-se dos requisitos de qualidade de serviço (Quality of Service - QoS) da VNF. Os pesquisadores enfrentam problemas para selecionar o eficiente e apropriado algoritmo de programação de recursos para uma carga de trabalho específica. Assim, partindo da observação de que o tráfego em um data center é uma mistura de componentes relativamente estáticos e rapidamente flutuantes, acreditamos que seja interessante aplicar estratégias de escalonador para ambos os componentes usando uma generalização do balanceamento de carga. Para tal, alguns dos desafios entrados são

- Como realizar escalonamento para executar cada função para minimizar o tempo de execução total sem degradar o desempenho do serviço e respeitando todas as precedências e dependências entre os VNFs que compõem a rede de substrato do data center?

- Como realizar escalonamento no data center de forma rigorosa com despesas de orçamento mínimo e aumenta o lucro global?

- Como reduzir o consumo de energia sem violação do SLA no escalonamento efetivo de recursos em data center?

- Como realizar um balanceamento eficiente da carga através do escalonamento das cargas de trabalho nos recursos disponíveis no data center?

- Como planejar um escalonamento robusto para serviços heterogêneos, considerando menos chances de falha não planejada em data center?

- Como reduzir o custo de execução e cumprir o prazo ao mesmo tempo na alocação de VNF no data center?

- Como reduzir o consumo de energia e seu impacto para o meio ambiente por meio de escalonamento no data center distribuído geograficamente? 
- Qual a melhor técnica de algoritmo exato ou heurístico que devem ser consideradas para um escalonamento eficiente dos recursos no data center?

- Como realizar o escalonamento de recursos de VNF dinamicamente no data center?

- Como desenvolver um algoritmo de escalonamento de recursos através da utilização eficaz de VNF e manutenção do SLA no data center?

- Qual o impacto de VNF na carga e distribuição de tráfego de rede entre os SFC sobre o desempenho do data center?

\subsection{Problemas de Falhas na Rede}

Observamos que outros pesquisadores realizaram pesquisas abordando problemas de falhas em sistemas, bem como em outras áreas relacionados à alocação de recurso ou ao encadeamento de serviço [Natella et al. 2016], [Endo et al. 2016], [Xie et al. 2016], [Herrera and Botero 2016], [Radjenović et al. 2013]. No entanto, nosso trabalho complementa esses estudos por ter uma abordagem diferenciada, pois, a partir de uma obervação desses trabalhos, juntamente com lacunas encontradas em alocação de NFV em data center, levantamos diversos problemas em aberto.

Dentro do conceito de dependabilidade [Avizienis et al. 2004] [Laprie 1992], encontram-se vários atributos relacionados à falha. A falha de uma VNF pode causar falha em outra funções subsequentes, levando a grande perda de dados que pode interromper serviços de comunicação. Neste artigo, levantamos desafios ainda não solucionados, também relacionados ao problema da capacidade de sobrevivência da rede em caso de falhas, o que pode causar graves interrupções do serviço devido às falhas em cascata e correlacionadas de vários nó(node) e links.

Falhas de redes relacionadas com alocação de recurso requerem abordagens sistemáticas, visando à melhoria da qualidade do sistema em termos de NFV aplicados ao data center. Para tal, listamos a seguir alguns desafios em aberto, seguindo as categorias de falhas de acordo com [Avizienis et al. 2004]:

- Como aplicar injeção de falha em VNF no data center?

- Qual a melhor técnica para predição de falha online de VNF no data center?

- Como planejar tolerância a falhas de SFC, quando as demandas de recursos podem mudar dependendo da carga de tráfego direcionada no data center?

- Qual a melhor técnica para detecção de falha em VNF no data center?

- Como planejar uma estratégia NFV tolerante a falhas de data center?

- Como executar provisionamento dinâmico de tolerância a falhas de NFV para data center?

- Como otimizar a utilização de VNF e minimizar o custo simultaneamente no data center?

- Como fazer previsão de defeitos, de forma eficiente, dos recursos em data center, a fim de reduzir o tempo de enfileiramento na fila da carga de trabalho?

- Como minimizar as pegadas de carbono e habilitar a capacidade dinâmica para manipulação e flutuação no provisionamento efetivo de recursos em nuvem?

- Como evitar, detectar e contornar bugs no projeto de hardware e software?

- Como gerenciar a altíssima complexidade dos sistemas atuais de computação construídos com dezenas de chips de milhões de transistores e com software de centenas de milhares de linhas de código? 
- Como conciliar alta confiabilidade e alta disponibilidade com as crescentes demandas por alto desempenho em NFV no data center?

- Como identificar e classificar as várias cargas de trabalho em data center?

- Como reconhecer claramente as presentes e as futuras falhas de VNF no data center?

- Como minimizar o tempo de execução das cargas de trabalho para melhorar a utilização dos recursos de VNF no data center?

- Como projetar uma única arquitetura que possa atender aos requisitos de QoS de NFV no data center?

- Como priorizar a fila de reescalonamento, que nem sempre é suficiente para erradicar o efeito de degradação de classes de serviço tolerantes a atrasos elevados de NFV no data center?

- Qual a melhor estratégia de escalonamento para promover menor tolerância de atraso de configuração, como priorizar a fila de reescalonamento, reservar uma fração dos recursos disponíveis e aumentar o espaço de pesquisa fornecendo alguns caminhos extras de VNF no data center?

\section{Considerações Finais}

Esta revisão da literatura foi conduzida com base no conhecimento atual do uso e desafios de alocação de recurso para NFV em arquiteturas de data center. Os estudos referentes ao uso da arquitetura NFV no data center estão relacionados a SFC, mapeamento, escalonamento e falhas da rede. A alocação de recurso de NFV em data center é um problema com muitos desafios a serem resolvidos nessa implementação. O problema da computação de soluções de alocação é NP-difícil. Por isso, existe uma forte demanda por algoritmos eficientes para resolver o problema. Assim, resumimos a pesquisa disponível com base em trabalhos disponíveis na web existente e apresentamos os desafios para pesquisa avançada que influenciam a disponibilidade, o desempenho e a eficiência energética dos recursos da rede do data center. Acreditamos que são grandes oportunidades de pesquisa no IETF para esta área. Vale a pena notar que há pouca preocupação da literatura considerando o mapeamento de SFC para o data center. Este é um ponto promissor para o trabalho futuro, uma vez que abordagens centralizadas sofrem com problema de escalabilidade e podem ser inviáveis em algumas situações, por exemplo, vários provedores.

\section{Referências}

Aldrin, S., Krishnan, R. R., Akiya, N., Pignataro, C., and Ghanwani, A. (2016). Service Function Chaining Operation, Administration and Maintenance Framework. InternetDraft draft-ietf-sfc-oam-framework-01, Internet Engineering Task Force. Work in Progress.

Amaldi, E., Coniglio, S., Koster, A. M., and Tieves, M. (2016). On the computational complexity of the virtual network embedding problem. Electronic Notes in Discrete Mathematics, 52:213-220.

Arregoces, M. and Portolani, M. (2003). Data center fundamentals. Cisco Press.

Avizienis, A., Laprie, J.-C., Randell, B., and Landwehr, C. (2004). Basic concepts and taxonomy of dependable and secure computing. IEEE transactions on dependable and secure computing, 1(1):11-33. 
Avramov, L. and Rapp (2016). Data Center Benchmarking Methodology. Internet-Draft draft-ietf-bmwg-dcbench-methodology-03, Internet Engineering Task Force. Work in Progress.

Callegati, F., Cerroni, W., and Contoli, C. (2016). Virtual networking performance in openstack platform for network function virtualization. Journal of Electrical and Computer Engineering, 2016.

Chiosi, M., Clarke, D., Willis, P., Reid, A., Feger, J., Bugenhagen, M., Khan, W., Fargano, M., Cui, C., Deng, H., et al. (2012). Network functions virtualisation: An introduction, benefits, enablers, challenges and call for action. In SDN and OpenFlow World Congress, pages 22-24.

Clayman, S., Maini, E., Galis, A., Manzalini, A., and Mazzocca, N. (2014). The dynamic placement of virtual network functions. In Network Operations and Management Symposium (NOMS), 2014 IEEE, pages 1-9. IEEE.

Do, T.-X. and Kim, Y.-H. (2017). High Availability Mechanisms for Service Function Chaining. Internet-Draft draft-xuan-nfvrg-ha-sfc-01, Internet Engineering Task Force. Work in Progress.

Endo, P. T., Rodrigues, M., Gonçalves, G. E., Kelner, J., Sadok, D. H., and Curescu, C. (2016). High availability in clouds: systematic review and research challenges. Journal of Cloud Computing, 5(1):16.

ETSI (2013). Network functions virtualisation (NFV)-virtual network functions architecture. Technical report, ETSI, Tech. Rep.

ETSI (2014). NFV performance \& portability best practises. ETSI Standard GS NFVPER, 1.

Fan, X., Weber, W.-D., and Barroso, L. A. (2007). Power provisioning for a warehousesized computer. SIGARCH Comput. Archit. News, 35(2):13-23.

Feamster, N., Rexford, J., and Zegura, E. (2013). The road to sdn. Queue, 11(12):20.

Herrera, J. G. and Botero, J. F. (2016). Resource allocation in nfv: A comprehensive survey. IEEE Transactions on Network and Service Management, 13(3):518-532.

Huang, P.-H., Li, K.-W., and Wen, C. H.-P. (2015). NACHOS: Network-aware chains orchestration selection for NFV in SDN datacenter. In Cloud Networking (CloudNet), 2015 IEEE 4th International Conference on, pages 205-208. IEEE.

IHS Markit (2015). NFV market to grow more than 5-fold through 2019, says ihs. http://www.infonetics.com/pr/2015/NFV-Market-Highlights.asp.

Kooli, M. and Di Natale, G. (2014). A survey on simulation-based fault injection tools for complex systems. In Design \& Technology of Integrated Systems In Nanoscale Era (DTIS), 2014 9th IEEE International Conference On, pages 1-6. IEEE.

Kumar, S., Tufail, M., Majee, S., Captari, C., and Homma, S. (2017). Service Function Chaining Use Cases In Data Centers. Internet-Draft draft-ietf-sfc-dc-use-cases-06, Internet Engineering Task Force. Work in Progress.

Laprie, J.-C. (1992). Dependability: Basic concepts and terminology. In Dependability: Basic Concepts and Terminology, pages 3-245. Springer. 
Liu, G. and Wood, T. (2015). Cloud-scale application performance monitoring with SDN and NFV. In Cloud Engineering (IC2E), 2015 IEEE International Conference on, pages 440-445. IEEE.

Maini, E. and Manzalini, A. (2014). Management and orchestration of virtualized network functions. In IFIP International Conference on Autonomous Infrastructure, Management and Security, pages 52-56. Springer.

McKeown, N., Mekkittikul, A., Anantharam, V., and Walrand, J. (1999). Achieving $100 \%$ throughput in an input-queued switch. IEEE Transactions on Communications, 47(8):1260-1267.

Miller, R. (2011). A look inside amazon's data centers. Data Center Knowledge.

Moens, H. and De Turck, F. (2014). VNF-P: A model for efficient placement of virtualized network functions. In Network and Service Management (CNSM), 2014 10th International Conference on, pages 418-423. IEEE.

Natella, R., Cotroneo, D., and Madeira, H. S. (2016). Assessing dependability with software fault injection: A survey. ACM Computing Surveys (CSUR), 48(3):44.

Ponemon Institute (2016). Cost of data center outages by emerson network power. http://files.server-rack-online.com/2016-Cost-of-Data-Center-Outages.pdf.

Quinn, P. and Halpern, J. M. (2014). Service Function Chaining (SFC) Architecture. Internet-Draft draft-quinn-sfc-arch-05, Internet Engineering Task Force. Work in Progress.

Radjenović, D., Heričko, M., Torkar, R., and Živkovič, A. (2013). Software fault prediction metrics: A systematic literature review. Information and Software Technology, 55(8):1397-1418.

Rankothge, W., Ma, J., Le, F., Russo, A., and Lobo, J. (2015). Towards making network function virtualization a cloud computing service. In Integrated Network Management (IM), 2015 IFIP/IEEE International Symposium on, pages 89-97. IEEE.

Riccobene, V., Lombardo, A., Manzalini, A., and Schembra, G. (2014). Network functions at the edge (netfate): design and implementation issues. National Telecommunications and Information Theory Group (GTTI).

Rosa, R. V., Santos, M. A. S., and Rothenberg, C. E. (2015). MD2-NFV: The case for multi-domain distributed network functions virtualization. In Networked Systems (NetSys), 2015 International Conference and Workshops on, pages 1-5. IEEE.

Salfner, F., Lenk, M., and Malek, M. (2010). A survey of online failure prediction methods. ACM Computing Surveys (CSUR), 42(3):10.

Samuel, K. and Rapp (2017). Considerations for Benchmarking Network Virtualization Platforms. Internet-Draft draft-skommu-bmwg-nvp-00, Internet Engineering Task Force. Work in Progress.

Schaffrath, G., Werle, C., Papadimitriou, P., Feldmann, A., Bless, R., Greenhalgh, A., Wundsam, A., Kind, M., Maennel, O., and Mathy, L. (2009). Network virtualization architecture: proposal and initial prototype. In Proceedings of the 1st ACM workshop on Virtualized infrastructure systems and architectures, pages 63-72. ACM. 
Shen, W., Yoshida, M., Kawabata, T., Minato, K., and Imajuku, W. (2014). vConductor: An NFV management solution for realizing end-to-end virtual network services. In Network Operations and Management Symposium (APNOMS), 2014 16th Asia-Pacific, pages 1-6. IEEE.

Singh, S. and Chana, I. (2016). Qos-aware autonomic resource management in cloud computing: a systematic review. ACM Computing Surveys (CSUR), 48(3):42.

Soares, J. and Sargento, S. (2015). Optimizing the embedding of virtualized cloud network infrastructures across multiple domains. In Communications (ICC), 2015 IEEE International Conference on, pages 442-447. IEEE.

Szabo, R., Figueira, N., and Lee, S. (2016). Policy-Based Resource Management. Internet-Draft draft-irtf-nfvrg-policy-based-resource-management-02, Internet Engineering Task Force. Work in Progress.

Vieira, M., Madeira, H., Irrera, I., and Malek, M. (2009). Fault injection for failure prediction methods validation. In 40th Int'l Conference on Dependable Systems and Networks (5th Workshop on Hot Topics in System Dependability).

Wang, R., Hu, H., and Yang, X. (2014). Potentials and challenges of C-RAN supporting multi-rats toward 5G mobile networks. IEEE Access, 2:1187-1195.

Xia, M., Shirazipour, M., Zhang, Y., Green, H., and Takacs, A. (2015). Optical service chaining for network function virtualization. IEEE Communications Magazine, 53(4):152-158.

Xie, Y., Liu, Z., Wang, S., and Wang, Y. (2016). Service function chaining resource allocation: A survey. arXiv preprint arXiv:1608.00095.

Zúñiga, J.-C., Bernardos, C. J., Rahman, A., Contreras, L. M., Lynch, P., and Aranda, P. A. (2017). Network Virtualization Research Challenges. Internet-Draft draft-irtf-nfvrggaps-network-virtualization-05, Internet Engineering Task Force. Work in Progress. 